DOI: https://doi.org/10.11144/Javeriana.upsy19.epbi

\title{
Estrategia de posicionamiento en bases internacionales de las revistas de psicología editadas y coeditadas por el Colegio Oficial de Psicólogos de Madrid*
}

\section{Positioning Strategy in International Databases of Psychology Journals Edited and Co-Edited by the Professional Association of Psychologists of Madrid}

\author{
MacArena Tortosa-PÉrez \\ Universidad Internacional de Valencia, España \\ ORCID: https://orcid.org/0000-0002-7900-4877 \\ Francisco González-Sala \\ Universidad de Valencia, España \\ ORCID: https://orcid.org/0000-0003-4124-7459 \\ María Constanza Aguilar-Bustamante \\ Universidad Santo Tomas, Colombia \\ ORCID: https://orcid.org/0000-0003-2990-9667 \\ Jesús Santolaya-Prego de Oliver \\ Universidad Europea de Valencia, España \\ ORCID: https://orcid.org/0000-0002-7930-4642
}

a Autor de correspondencia. Correo electrónico: macarena.tortosap@campusviu.es

Para citar este artículo: Tortosa-Pérez, M., GonzálezSala, F., Aguilar-Bustamante, M. C., \& SantolayaPrego de Oliver, J. (2020). Estrategia de posicionamiento en bases internacionales de las revistas de psicología editadas y coeditadas por el Colegio Oficial de Psicólogos de Madrid. Universitas Psychologica, 19, 1-16. https://doi.org/10.11144/Javeri ana.upsy19.epbi

\section{RESUMEN}

La presencia de colegios profesionales, universidades y fundaciones como editoriales es una característica de las revistas iberoamericanas de psicología indexadas en el Journal Citation Reports (JCR). Este trabajo analiza, desde una perspectiva sociobibliométrica, la estrategia de posicionamiento seguida por el Colegio Oficial de Psicólogos de Madrid (COP Madrid) en relación con las revistas de psicología que edita. Los resultados indican un aumento de las revistas coeditadas y editadas por el Colegio en los últimos años, siete en JCR y ocho en SCImago Journal Rank (SJR), y una mayor visibilidad al darse un aumento en el número de citas, de visitas y de descargas de los artículos publicados en ellas. Se puede concluir que la estrategia seguida por el COP de Madrid unificando las normas de edición de todas sus revistas, la profesionalización de la gestión editorial, la edición electrónica, de acceso abierto y la inclusión en bases de datos internacionales ha llevado a este a ser el principal grupo editorial de revistas de psicología en JCR y SJR en España e Iberoamérica, si bien se requiere una estrategia más amplia que contemple la igualdad de género en los equipos directivos.

Palabras clave

revistas de psicología; JCR; Colegio de Psicólogos de Madrid; estrategia editorial; posicionamiento internacional; cuartiles. 


\begin{abstract}
The Latin American journals of psychology indexed in the Journal of Citation Reports (JCR), are generally edited by professional associations of psychologists, universities and private foundations. This paper analyzes the positioning strategy adopted by the Professional Association of Psychologists of Madrid, regarding the psychological journals in the area in which they edit. The methodological approach taken to explore the topic uses socio-bibliometric techniques gathering numerical data, and then using that data to perform analyses for a well-informed description of what the topic might be. The results reveal that journals, whose editor or co-editor was Professional Association of Psychologists of Madrid, have increased their visibility in recent years, in both, the SCImago Journal Rank (SJR $=8$ journals) and that of the Journal of Citation Reports (JCR $=7$ journals) with a greater citation count for its articles, and a higher number of views and downloads for the papers published in its journals. The conclusion is, that the positioning strategy adopted by the Professional Association of Psychologists of Madrid was an effective one, involving the unification of certain rules relating to the edition of articles in their journals, the professionalization of editorial management, the electronic publishing with digital publication of journals and using the open access publishing model for scholarly communication that makes research information available to readers at no cost, as well as their integration in the list of international databases. Although a broader strategy concerning gender equality is recommended, the Spanish professional association of psychologists still stands today as the main editorial group of psychological journals indexed in JCR and SJR of Spain

Keywords

psychology journals; Journal of Citation Reports; Professional Association of Psychologists of Madrid; editorial strategy; international positioning; quartiles.
\end{abstract}

Las revistas científicas definen uno de los canales de comunicación formal de descubrimientos científicos y de difusión de conocimientos de interés para la comunidad científica. No es el único, pero quizás hoy si sea el más utilizado a la hora de dar a conocer los conocimientos, convirtiendo algo privado en público, ya que permiten que la comunidad científica comparta y se apropie de contenidos de investigación y reflexión que desconocía. La publicación en revistas de mayor impacto posibilita una mayor visibilidad a los trabajos, lo cual revierte en un mayor número de citas, otorgando estatus y jerarquía a los investigadores.
En el caso de la psicología española, tal y como apuntan Jiménez-Contreras, RobinsonGarcía y Cabezas-Clavijo (2011), las agencias de evaluación españolas, como la Agencia Nacional de Evaluación y Prospectiva (ANEP), la Comisión Nacional Evaluadora de la Actividad Investigadora (CNEAI) y la Agencia Nacional de Evaluación de la Calidad y Acreditación (ANECA) otorgan un papel prioritario en la evaluación de acreditaciones y de las carreras investigadoras a las publicaciones en revistas de la Web of Science (WoS) y al factor de impacto. Pero, la presencia de revistas indexadas en la WoS por país es muy desigual. Así pues, entre los años 2014 a 2016 el 80 \% de las revistas en esta base de datos eran editadas en Estados Unidos y en el Reino Unido, habiendo únicamente 11 países con revistas en el primer cuartil del Journal Citation Reports (JCR) (Osca-Lluch, GonzálezSala, Haba-Osca, Tortosa, \& Peñaranda-Ortega, 2019). A esta situación de desigualdad, hay que añadir las características diferenciales entre las revistas editadas en unos y otros países. En este sentido, González-Sala, Osca-Lluch y Haba-Osca (2019) encuentran que, entre las revistas iberoamericanas y holandesas de psicología en el JCR, llama la atención una mayor presencia de colegios profesionales, fundaciones $\mathrm{o}$ asociaciones y universidades como editoriales de las publicaciones iberoamericanas, frente a la presencia en las revistas holandesas de grandes grupos editoriales (tales como Elsevier, Springer, Taylor and Francis, entre otras), los cuales son responsables del $70 \%$ de las publicaciones en las categorías del Social Science Citation Index (SSCI) (Larivière, Haustein, \& Mongeon, 2015).

Las publicaciones iberoamericanas de psicología experimentan un aumento con los años (Osca-Lluch, González-Sala, Fonseca, \& Civera, 2017), y en el caso concreto de las revistas españolas de psicología se pasa de tener una revista en 2001 en JCR a 15 revistas en el 2017 (Tortosa-Pérez, Osca-Lluch, Alfaro-Ferreres, \& López-López, 2019). En este aumento, destaca el papel clave que juega la estructura colegial, y muy especialmente el Colegio Oficial de Psicólogos de Madrid (COP Madrid, en adelante), al ser responsable de más de la mitad de las 
revistas españolas de psicología, incluidas en alguna categoría del JCR (Science Citation Index Expanded [SCIE] y Social Science Citation Index [SSCI]) y del Scimago Journal Ranking (SJR) (Tortosa-Pérez, González-Sala, SantolayaPrego de Oliver, \& Aguilar-Bustamante, 2020).

Partiendo de los estudios sociales de la ciencia que pretenden determinar en qué grado determinados factores sociales, institucionales, económicos, políticos o culturales influyen en el propio desarrollo de esta (Bucchi, 2004; KnorrCetina \& Mulkay, 1983), tiene sentido analizar el papel que juegan las instituciones en la ciencia a través de la edición de revistas científicas indexadas en bases de datos internacionales y, en concreto en el caso de las revistas españolas de psicología, el papel que ha jugado el COP Madrid. La andadura del Colegio Oficial de Psicólogos se inicia en 1980, Delegación de Madrid, nombre que mantendrá durante 20 años. En ese momento, acababa de aprobarse prácticamente por unanimidad, en el Congreso y el Senado la Ley 43/1979 de 31 de diciembre, de creación del Colegio Oficial de Psicólogos (Jefatura del Estado, 1980), como un paso más del reconocimiento profesional de la psicología en España, pero será, en 2001, cuando se constituirá el COP Madrid mediante el Decreto 1/2001 de 11 de enero de la Consejería de Presidencia de la Comunidad de Madrid.

En este marco, se plantea como objetivo del presente trabajo estudiar la estrategia de visibilización y posicionamiento en bases de datos internacionales de revistas de psicología seguida por parte del COP Madrid, desde una metodología sociobibliométrica.

\section{Método}

Procedimiento y materiales

Partiendo de una perspectiva sociobiliométrica (Carpintero, 1980, 1983a, 1983b; Carpintero \& Peiró, 1981), se realizó un análisis del proceso de formación, desarrollo y posicionamiento del COP Madrid en relación con las revistas editadas por este. Posteriormente, con respecto a estas publicaciones se analizaron diferentes indicadores bibliométricos tales como: el número de revistas españolas de psicología en JCR entre 2000 y 2018, la editorial, el cuartil en el JCR (SSCI) y el SJR, la circulación en bases de datos nacionales e internacionales, el número de citas, el número y procedencia de visitas y de descargas de artículos y el sexo de los miembros del equipo editorial.

Los materiales analizados fueron las memorias del COP Madrid y las ocho revistas editadas y coeditadas por el Colegio: Clínica y Salud, Journal of Work and Organizational Psychology, Psychosocial Intervention, Anuario de Psicología Jurídica, Psicología Educativa, European Journal of Psychology Applied to Legal Context, Clínica Contemporánea .Revista de Psicología Aplicada al Deporte y al Ejercicio Físico.

\section{Resultados}

Desarrollo del COP Madrid y de las revistas editadas por el Colegio de Psicólogos de Madrid

La Delegación del Colegio de Psicólogos de Madrid como editor de revistas: fundación y desarrollo

Tras su creación en 1980 y por sugerencia parlamentaria, la Ley 43/1979 contempló la creación de un único colegio estatal, integrado por delegaciones, con vocación, recogida en los estatutos provisionales, de segregación de esas delegaciones para convertirse en colegios autonómicos (Hernández-Gordillo, 1989). La Disposición Adicional Primera de la Ley establece que la Coordinadora Estatal de Secciones de Psicólogos se convierte en Comisión Gestora del Colegio, con la misión de elaborar unos estatutos provisionales.

El 14 de abril de 1980, se promulgan los estatutos provisionales del Colegio Oficial de Psicólogos (Ministerio de Universidades e Investigación, 1980), donde se reconoce a dicha Comisión Gestora como Junta Provisional de Gobierno. A su amparo, el 5 de mayo, se celebran las elecciones para la primera Junta de Gobierno 
del Colegio, que tendría como decano a Carlos Camarero (1945-2001), presidente también de la Comisión Gestora de la Delegación de Madrid.

Por razones de distribución territorial, y dada la falta de secciones de psicólogos en muchos Colegios de Licenciados que estuvieran funcionando en aquel momento (apenas 20), la Delegación de Madrid arranca como una macrodelegación que abarca las provincias de Madrid, Toledo, Guadalajara, Cuenca, Ciudad Real, Albacete, Murcia, Cáceres y Badajoz. Una Delegación que, además, debía atender a la Secretaría Estatal del Colegio, que radicaba en Madrid. Una situación que, en la práctica, llevaba a que los objetivos de la Delegación se confundiesen con los generales del Colegio (Junta de Gobierno, 1981). Comenzaba "la década del psicólogo, de la definición de su papel social" (Camarero, 1989, p. 67). Son años cuando la consolidación institucional y la definición del papel social y los perfiles profesionales de los psicólogos definían los grandes objetivos por alcanzar.

El Colegio, sin duda un colegio con historia (COP Madrid, 2019a; Martínez, 2001), mantiene, desde su creación, una serie de líneas de trabajo destinadas a potenciar la promoción y el desarrollo técnico y científico de la profesión (Santolaya, 2001). Entre esas líneas de trabajo, junto a la realización de eventos de carácter científico y cursos de formación y actualización profesional, destaca el desarrollo de un modelo organizativo por sectores profesionales (Secciones) y la creación de publicaciones, periódicas y no periódicas (Civera \& Alonso, 1995; Civera \& Tejero, 1993).

En julio de 1981, como número 0, comienza la primera revista. "Este es el primer Boletín que presentamos con el nombre de "Papeles del Colegio" desde que nuestra Delegación comenzó su funcionamiento" (Junta de Gobierno, 1981b, p. 1). Le seguiría otro número en diciembre. Aunque lo prepara y edita la Delegación de Madrid, tiene proyección estatal, se distribuye en todas las Delegaciones que lo solicitan (COP Madrid, 1982).

Cuatro años después, en 1985, la Delegación impulsaría, con ayuda de la Sociedad Española de Psicología, la Revista de Psicología del Trabajo $y$ de las Organizaciones, incorporando el título en inglés en 2009, siendo este el que se usa en la actualidad. Esta revista es financiada inicialmente con los fondos remanentes del I Congreso de Psicología del Trabajo (Madrid, 9 de abril de 1983) (Munduate, 1985). Diez años después, se reafirma el carácter colegial de la revista, ni comercial ni universitario, señalando que uno de sus principales objetivos es "la formación de los profesionales y actuar de foro de discusión para la puesta en común de experiencias y de reflexiones de los psicólogos que trabajan en el ámbito" (Hernández-Gordillo, 1995, p. 7).

Mientras, en 1982, se creaba el servicio de documentación, y se iniciaba la publicación decana de la entonces Delegación, la Guía del Psicólogo. En 1986, publica unas Selecciones de Prensa que recogía noticias y artículos relevantes relacionados con la psicología y documentación psicológica, que ofrecía información de referencia sobre documentos psicológicos elaborados y/o publicados en España y sobre documentación especializada recibida en las principales bibliotecas.

En 1990, al tiempo que se establecía el título oficial de Licenciado en Psicología, aparece la revista Clínica y Salud, revista de psicología clínica y salud. Continúa desarrollándose el proyecto colectivo orientado a la definición de una identidad profesional y el logro de un necesario protagonismo social. "En la idea de que los sectores tengan sus vehículos propios de expresión hemos visto como surgían publicaciones como "Selecciones de Prensa", "Clínica y Salud" y próximamente aparecerá un "Anuario de Psicología Jurídica", y con toda seguridad revistas sectoriales para las áreas de Servicios Sociales y Psicología Educativa" (Junta General Delegación de Madrid, 1991, p. 4). En esta línea, en 1991, se edita el Anuario de Psicología Jurídica (Ramírez, 1991), Intervención Psicosocial (Casas, 1992), revistas que, en 2009, incorporaron el título en inglés, siendo este el que se emplea en la actualidad. En 1995, surge Psicología Educativa (Anónimo, 1995). A 
todas ellas se accede por suscripción personal o institucional.

Esta evolución se ha debido en gran parte al desarrollo de las comisiones profesionales, primero y después a las Secciones de la Delegación. La sección es la unidad estructural básica del Colegio, que se crean para organizar las actividades sectoriales en los distintos campos de intervención psicológica y aspiran a delimitar el rol mediante una revista que sea representativa.

En 1996, siguiendo con la activa labor de posicionamiento de la psicología y la Delegación en los medios de comunicación y en los ámbitos de referencia, el Departamento de Publicaciones del COP de Madrid realiza diferentes gestiones orientadas a la mejora y promoción de estas publicaciones: presentación y promoción de las revistas en congresos y jornadas, realización de diferentes mailings para captar suscripciones, unificación de los criterios de edición y promoción de las revistas en otras publicaciones y en las universidades, solicitud de incorporación a las bases de datos nacionales e internacionales de mayor prestigio (COP de Madrid, 1997, p. 35) y, desde 2001, publicidad de las publicaciones en diferentes medios (COP Madrid, 2002, p. 67).

En 1997, comienza a editarse, en formato CD-Rom, una base de datos bibliográfica especializada en psicología y disciplinas afines, elaborada en colaboración con la biblioteca de la Facultad de Psicología de la Universidad Complutense de Madrid. El recurso tuvo un gran éxito inmediato, tanto institucionalmente -fue adquirido por numerosas universidades de habla española- como entre colegiados, investigadores y profesionales afines (COP Madrid, 1998, p. 15). Ya en la siguiente edición, incluye la edición electrónica, a modo de muestra de un número de cada una de las cinco revistas editadas por la Delegación del Colegio.

Se favorece el consumo interno de las publicaciones. La Memoria de 1998 recoge que los colegiados podrán suscribirse gratuitamente a la revista especializada que prefieran de entre las que se editan (COP Madrid, 1999, p. 13) y la edición de 1999 de PSICODOC incluye la sección "Revistas", edición electrónica de las cinco revistas editadas.

Además, continúa el proceso de posicionamiento e internacionalización. Las bases de datos PsychoINFO, PsyLIST, ClinPSYC y Psychological Abstracts, recogen Clínica y Salud, a la que un año después se uniría la Revista de Psicología del Trabajo y las Organizaciones (COP Madrid, 2000). No solo esas dos, sino todas se incluían también en PSICODOC, PSEDISOCCINDOC, IBECS, IN-RECS, etc.

Este primer período se cierra cuando la Junta de Gobierno del Colegio Oficial de Psicólogos, de conformidad con lo establecido en el artículo 21 de sus estatutos generales (Ministerio de Educación y Cultura, 1999), adoptó el acuerdo para la segregación de sus Delegaciones territoriales (Ministerio de Educación, Cultura y Deporte, 2000), segregación que se produjo mediante el Decreto 1/2001, de 11 de enero de la Consejería de Presidencia de la Comunidad de Madrid. Nacía el Colegio Oficial de Psicólogos de Madrid. En la Asamblea Constituyente del 8 de mayo de 2001, se constituyó\# el Colegio Oficial de Psicólogos de Madrid, y se aprobaron los estatutos que regulan su funcionamiento.

\section{El Colegio de Psicólogos de Madrid como editor de revistas con factor de impacto}

En 2003, las revistas se reseñan en 19 nuevas bases de datos internacionales a través de un distribuidor multinacional de información electrónica. El objetivo fue visibilizar internacionalmente "lo que se está haciendo desde España en las diferentes áreas de la Psicología" (COP Madrid, 2004, p. 54).

Nace también el Departamento de Tecnologías de la Información para dar soporte a las necesidades que, en materia de avances informáticos, tuviese el Colegio y para desarrollar proyectos de futuro, entre otros, la configuración de una nueva web corporativa, que se haría pública en abril de 2007, y el impulso de servicios integrales de gestión de las revistas del Colegio, que ya entonces se editan electrónicamente (COP Madrid, 2007). A través de la web, se 
pretendía favorecer la circulación y consumo de las revistas. De hecho, desde septiembre de 2008, los colegiados de Madrid tuvieron acceso online gratuito al texto completo de las revistas que edita el COP Madrid, que casi inmediatamente se convertiría en el área con mayor número de accesos.

Durante los 23 años transcurridos desde que se iniciara (1985) la entonces Revista de Psicología del Trabajo y de las Organizaciones, la gestión editorial de cada una de las revistas se realizaba manualmente a través del correo postal y correo electrónico. A partir de 2008, las revistas comenzarán a gestionarse a través de una plataforma de contenidos desde la que se controlaba todo el proceso editorial. El nuevo modelo, además de agilizar el proceso editorial, garantizaba la calidad de la gestión y mejoraba el repositorio, ya que todos los artículos, revisiones y notificaciones se tramitan mediante la nueva plataforma.

Mientras tanto, las políticas de Estado pormenorizaban y definían los criterios específicos de evaluación de la actividad científica en el campo de las ciencias del comportamiento, que muy pocas revistas españolas cumplían, fruto de la limitada profesionalización de la gestión editorial y de su ausencia de las bases de datos internacionales, por lo que su protagonismo internacional era escaso (Giménez, Rodríguez, \& de la Moneda, 2009; Ruiz, Delgado, \& Jiménez, 2006).

Todos aquellos criterios confluyeron sobre la necesidad de publicar en revistas con factor de impacto, iniciándose así lo que dio en llamarse un "impact factor style of thinking” (Fernández \& Rodríguez, 2014). Para afrontar la situación, la administración hizo un gran esfuerzo didáctico, a través de la Fundación Española para la Ciencia y la Tecnología (FECYT), por difundir los estándares internacionales de edición. Universidades, fundaciones, asociaciones y colegios fueron receptivos, comenzaron a transformar o crear revistas que cumplieran los rigurosos criterios necesarios para ser admitidas en el JCR, el índice de citas de referencia.
En 2008, había cinco revistas de psicología en JCR/SSCI, ninguna de ellas del COP Madrid, donde el papel y el español eran los soportes de información dominantes. A pesar de su elevado impacto dentro de España (Pérez, 2008), la primera presencia del COP Madrid en JCR sería en 2011 a través de la coedición, con la Facultad de Psicología de la Universidad Complutense de Madrid, de una revista universitaria, editada íntegramente en inglés, Spanish Journal of Psychology, recogida en la base de datos de citas desde 2007. Reproduciría el modelo con la revista European Journal of Psychology Applied to Legal Context, recogida en JCR desde 2012 y editada por la Sociedad Española de Psicología Jurídica y Forense y la Asociación Iberoamericana de Justicia Terapéutica (Tortosa et al., 2019).

En 2018, son 17 las revistas de psicología en JCR/SSCI, las cinco últimas en incorporarse son del COP Madrid. En 2017, Clínica y Salud, Journal of Work and Organizational Psychology . Psychosocial Intervention y en 2018, Anuario de Psicología Jurídica y Psicología Educativa. Todas en formato electrónico, algunas solo en inglés, otras preferentemente en español, pero aceptando originales en inglés, y algunas publicando en español o inglés, según la lengua del documento original.

El COP Madrid desarrolló un plan estratégico de publicaciones para incrementar la calidad científica y mejorar los procesos de gestión editorial de sus revistas, con la intención de asaltar las bases de datos internacionales de referencia. Para ello, creó un Comité asesor compuesto por los directores de las revistas, por miembros de la Junta de Gobierno y por técnicos del Colegio (Colegio Oficial de Psicólogos de Madrid, 2010, p. 5).

El proceso se inicia con la renovación de los equipos editoriales entre 2009 y 2013. Los nuevos equipos siguieron las recomendaciones del Comité, que, tras analizar las debilidades de las revistas, impulsó una política editorial común. Se consensuaron aspectos estratégicos comunes de funcionamiento (orientación aplicada vs. académica, propiedad intelectual, incorporación del código Digital Object Identifier (DOI), difusión, libertad editorial, etc.), se normalizó la 
periodicidad en la publicación de las revistas, se establecieron criterios compartidos para la evaluación de manuscritos, se incrementó el número de originales en inglés, se configuraron sistemas de calidad y control, auditorías y procesos de autoevaluación de las revistas, se establecieron los principios éticos y deontológicos de la política editorial, se impulsaron y establecieron las prioridades de las medidas por adoptar en el calendario anual que facilitara la incorporación a directorios y plataformas iberoamericanas y de otros entornos geopolíticos (COP de Madrid, 2010; 2011; 2012; 2013; 2014), una política que propiciará un progresivo posicionamiento internacional de sus revistas.

A las cinco revistas que venía editando en solitario, se unen, como revistas exclusivamente en formato online a través de la página web, en 2010, Clínica Contemporánea. Revista de Diagnóstico Psicológico, Psicoterapia y Salud, "con un compromiso férreo con la profesión" (Aznar \& Brox, 2010, p. 3) y, en 2016, la Revista de Psicología Aplicada al Deporte y el Ejercicio Físico, que pretende abrir una puerta "a la comunidad científica y a los diferentes profesionales del ámbito del deporte" para difundir en español sus contribuciones (García-Naveira, 2016, p. 1).

Pronto se notará la profesionalización de la gestión editorial. En el año 2012, se comienzan a publicar artículos en pre-press. Además,

[...] la Fundación para la Ciencia y la Tecnología (FECYT) reconoció\# como excelentes a 2 de las revistas editadas por el COP Madrid, en concreto, Revista de Psicología del Trabajo y de las Organizaciones y Psychosocial Intervention. Únicamente 27 de las 255 publicaciones presentadas lograron la citada catalogación, y las editadas por el Colegio fueron las dos únicas que lo consiguieron en el ámbito de la Psicología. (COP de Madrid, 2013, p. 21)

En 2014, se sumarían las revistas Clínica y Salud, European Journal of Psychology Applied to Legal Context, Psicología Educativa, Spanish Journal of Psychology y dos años después, en 2016, el Anuario de Psicología Jurídica. Además, la coedición de la Revista de Historia de la Psicología y Ansiedad y Estrés, desde 2016, permitía sumar dos revistas más a este selecto grupo, ya que tenían el sello vigente al haberlo obtenido en la cuarta convocatoria: una distinción que irían renovando en ediciones sucesivas.

En febrero de 2013, el COP Madrid firmó un acuerdo de colaboración con la editorial Elsevier España, con el objetivo de desarrollar la producción de cinco publicaciones (Revista de Psicología del Trabajo y de las Organizaciones, Psychosocial Intervention, Clínica y Salud, Psicologi\# a Educativa y Anuario de Psicología Jurídica), y de la European Journal of Psychology Applied to Legal Context, aunque el COP Madrid continuará ocupándose de la edición. Elsevier proporciona una nueva plataforma de gestión de contenidos, y pone a disposición de las revistas un experimentado equipo profesional. El nuevo sistema automatizado de producción (PTS) integra edición en papel e internet, lo que permite unificar la producción y la transformación paralela de los manuscritos. Durante el inicio de esta relación comercial (2013-2014), culmina el proceso de cambio y unificación de imagen (maquetación de portada, presentación y contenido de los artículos), que se iniciara en 2009 con Intervención Psicosocial. Se acababa de construir una nueva línea e imagen editorial única de las revistas del COP Madrid.

Aquel 2013, el Colegio incorporaba el Código de Conducta (COPE) para editores de revistas, y acababa de actualizar las normas editoriales, unificándolas en todas las revistas. Son años en los que dejan de publicarse solo originales escritos en español, para aceptarse artículos tanto en español como en inglés, además, en los originales en español debe incluirse un extended summary (hasta 2000 palabras) en inglés.

El objetivo del acuerdo, que suponía una elevada inversión, era doble, mejorar los procesos editoriales, y la visibilidad e internacionalización de las revistas que editaba. Un objetivo que se alcanzó pronto. En 2014, tres de las revistas que edita el COP Madrid (Clínica y Salud, Psicología Educativa y Anuario de Psicología Jurídica) venían a sumarse al Journal of Work and Organizational Psychology y a Psychosocial Intervention en la base de referencias bibliográficas y citas Scopus, donde 
también estaban European Journal of Psychology Applied to Legal Context y Spanish Journal of Psychology. Cuatro años después, el proceso ha continuado, son siete de las 12 revistas editadas por el COP Madrid las incluidas en esta prestigiosa base de datos internacional. Los indicadores cuantitativos de calidad permiten comparaciones, y se aproximan a las demandas españolas de la Agencia de Evaluación. Otro fruto inmediato propiciado por los cambios en la gestión y la política editorial, iniciados en 2009, fue el reconocimiento por parte de la Fundación para la FECYT, de todas las revistas editadas o coeditadas hasta entonces por el COP Madrid, como excelentes.

En 2016, se integran en la web los contenidos históricos de las revistas (anteriores al 2015), mejorando así de manera importante los recursos documentales a disposición de quienes accedían a la página, colegiados o no (COP Madrid, 2017, p. 19). Además, continuaba la destacable política de coedición que iniciara en 2011 y 2012. A los dos casos ya mencionados, se añade la edición, en 2014, de la Revista de Mediación del Instituto Motivacional Estratégico (IMOTIVA), producida por el Instituto y coeditada también con la Universidad Carlos III de Madrid. En 2016, el COP Madrid editará la Revista de Historia de la Psicología, órgano de expresión de la Sociedad Española de Historia de la Psicología y producida por esta, y Ansiedad y Estrés, voz de la Sociedad Española para el Estudio de la Ansiedad y el Estrés, productora de la misma.

Así, en 2016, el COP Madrid edita o coedita un total de 13 revistas cienti\#ficoprofesionales. Siete íntegramente (Cli\#nica y Salud, Journal of Work and Organizational Psychology/Revista de Psicología del Trabajo y de las Organizaciones, Psychosocial Intervention/ Intervención Psicosocial, Clínica Contemporánea, Psicología Educativa, Anuario de Psicología Jurídica y Revista Aplicada al Deporte y al Ejercicio Físico), y cinco (Spanish Journal of Psychology, European Journal of Psychology Applied to Legal Context, Revista de Mediacio\#n, Revista de Historia de la Psicologi\#a. Ansiedad y Estre\#s) en coedición con otras instituciones.
En 2016 y 2017, se cierra con un notable éxito el Plan estratégico de Publicaciones del Consejo Asesor de Revistas. Tres revistas más (Journal of Work and Organizational Psychology, Cli\#nica y Salud y Psychosocial Intervention) se incorporan en la edición de 2017 del JCR/SSCI, y otras dos (Anuario de Psicología Jurídica y Psicología Educativa) en la de 2018. En total siete (41.18 \%) de las 17 revistas españolas de psicología recogidas en JCR/SSCI son editadas o coeditadas por el COP Madrid. Además, cuatro (Ansiedad y Estrés, Revista de Mediación, Revista de Historia de la Psicología . Clínica Contemporánea) de las otras cinco revistas que edita y coedita están también en la WOS, en este caso en el Emerging Source Citation Index.

\section{Indicadores bibliométricos de las revistas de psicología editadas por el COP de Madrid}

Distribución de revistas españolas de psicología en JCR(SSCI) entre 2000-2018

En la Figura 1, puede observarse el crecimiento de las revistas españolas de psicología en JCR, desde el año 2000, pudiéndose comprobar como este aumento ha ido acompañado por la inclusión de las revistas editadas por el COP de Madrid, principalmente en los años 2017 y 2018.

\section{Figura 1}

Crecimiento por año del número revistas psicología españolas en JCR/SSCI.

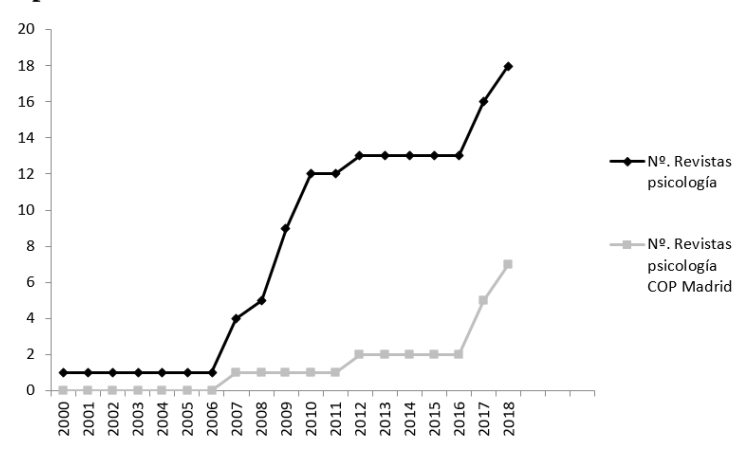


Posición en el JCR/SSCI y SJR de las revistas editadas por el COP de Madrid

Atendiendo al cuartil que ocupan las revistas del COP de Madrid en JCR y SJR, tres de ellas se encuentran entre los dos primeros cuartiles, pudiéndose observar un mejor posicionamiento en el JCR y SJR con los años (Tablas 1 y 2). De hecho, todas sus revistas, excepto una, la Revista de Psicología Aplicada al Deporte y el Ejercicio Físico, están en la Web of Science, siete en JCR/ SSCI y cuatro en ESCI. A su vez, las siete revistas que están en JCR/SSCI también se incluyen en SJR. En definitiva, un grupo de revistas en general muy apetecibles para publicar en ellas.

\section{Tabla 1}

Distribución cronológica según cuartiles en JCR/ SSCI de las revistas del COP Madrid

\begin{tabular}{|c|c|c|c|c|c|c|c|c|}
\hline Revistas & 2011 & 2012 & 2013 & 2014 & 2015 & 2016 & 2017 & 2018 \\
\hline $\begin{array}{l}\text { Anuario de Psicologia } \\
\text { Jurídica }\end{array}$ & & & & & & & & $\mathrm{Q4}$ \\
\hline Clinica y Salud & & & & & & & Q4 & Q4 \\
\hline $\begin{array}{l}\text { European Journal of } \\
\text { Psychology Applied to Legal } \\
\text { Context }\end{array}$ & & Q3 & Q3 & Q2 & Q3 & Q2 & Q1 & Q1 \\
\hline $\begin{array}{l}\text { Journal of Work \& } \\
\text { Organizational Psychology }\end{array}$ & & & & & & & Q3 & Q3 \\
\hline & & & & & & & & Q2 \\
\hline Psych & & & & & & & Q2 & Q1 \\
\hline Spanish Journal Psychology & Q2 & Q2 & Q2 & Q3 & Q3 & Q3 & Q3 & Q3 \\
\hline
\end{tabular}

\section{Tabla 2}

Distribución cronológica según cuartiles en SJR de las revistas del COP Madrid

\begin{tabular}{|c|c|c|c|c|c|c|c|c|}
\hline Revistas & 2011 & 2012 & 2013 & 2014 & 2015 & 2016 & 2017 & 2018 \\
\hline Anuario Psicología Juridica & & & & Q4 & $4 \quad$ Q4 & Q4 & Q3 & Q4 \\
\hline Clinica y Salud & & & & Q3 & Q3 & Q4 & Q4 & Q3 \\
\hline $\begin{array}{l}\text { European Journal of } \\
\text { Psychology Applied to } \\
\text { Legal Context }\end{array}$ & Q3 & Q2 & Q3 & Q3 & Q3 & Q2 & Q2 & Q1 \\
\hline $\begin{array}{l}\text { Journal of Work \& } \\
\text { Organizational Psychology }\end{array}$ & & Q4 & Q4 & Q4 & Q4 & Q3 & Q3 & Q2 \\
\hline Psicología Educativa & & & & Q4 & Q4 & Q4 & Q3 & Q3 \\
\hline Psychosocial Intervention & & Q3 & Q3 & Q3 & Q3 & Q3 & Q3 & Q2 \\
\hline $\begin{array}{l}\text { *Spanish Journal of } \\
\text { Psychology }\end{array}$ & Q2 & Q2 & Q2 & Q3 & Q3 & Q3 & Q3 & Q3 \\
\hline *Ansiedad y Estrés & & & & & & Q4 & Q4 & Q4 \\
\hline
\end{tabular}

(*)Spanish Journal of Psychology, convenio coedición 2011; European Journal of Psychology Applied to Legal Context, convenio coedición 2012; Ansiedad y Estrés, convenio coedición, 2016; Revista de Historia de la Psicología, convenio coedición 2016
Circulación nacional e internacional de las revistas del COP Madrid

Son revistas que, además, mantienen un elevado grado de circulación nacional e internacional. Circulan, con diferente protagonismo, por más de 40 bases de datos. En la Tabla 3 se ofrece, para 2018, las bases de datos, catálogos y directorios de las revistas editadas por el COP Madrid.

El Anuario de Psicología Jurídica, Clínica y Salud, Journal of Work and Organizational Psychology, Psicología Educativa. Psychosocial Intervention son las revistas que más difunden sus contenidos de forma simultánea; están a su vez entre las más difundidas, que forman parte del grupo de revistas top españolas, las 17 con factor de impacto en SJR y JCR/SSCI (Tortosa-Pérez et al., 2019).

\section{Tabla 3}

Difusión de las revistas del COP Madrid en bases de datos nacionales e internacionales

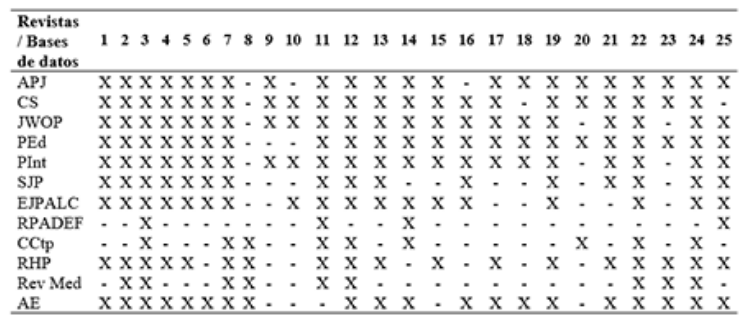

Nota. Bases de datos (1.ÍnDICEs CSIC; 2.DIALNET; 3.PSICODOC; 4.RESH; 5.PSYCINFO; 6.SCOPUS; 7.WoS; 8.ESCI; 9.REDALYC; 10.SCIELO; 11 DOAJ; 12.LATINDEX; 13.SELLO CALIDAD FECYT; 14.EBSCO; 15.Google Académico; 16.IBECS; 17.Psyke; 18.ScienceDirect; 19.CARHUS; 20.ELibro; 21.IN RECS; 22.MIAR; 23.RebIUn.

24.CIRC. 25.ERIH PLUS). Revistas: APJ (Anuario de Psicología Jurídica); CS (Clínica y Salud); JWOP (Journal of Work \& Organizational Psychology); PEd (Psicología Educativa); PInt (Psychosocial Intervention); SJP (Spanish Journal of Psychology); EJPALC (European Journal of Psychology Applied to Legal Context); RPADEF (Revista de Psicología Aplicada al Deporte y al Ejercicio Físico); CCtp (Clínica Contemporánea ); RHP (Revista de Historia de la Psicología); Rev Med (Revista de Mediación); AE (Ansiedad y Estrés). 
Distribución por sexo en los órganos directivos de las revistas de psicología en JCR editadas por el COP Madrid

En la Tabla 4, se recogen datos relativos a los equipos editoriales de las revistas del COP Madrid, en función de su sexo. Son 358 los nombres encontrados, alguno repetido en más de una revista. Aparece la habitual distribución asimétrica en puestos directivos, dos tercios de los integrantes son hombres, siendo la diferencia especialmente notoria en el caso de la figura principal, seis directores $(85.71 \%)$ y una sola directora (14.29\%), resultados similares a los encontrados por González-Sala y Osca-Lluch (2018).

Los datos parecen reflejar más la realidad universitaria que la realidad profesional, en la que predominaban, y predominan, las mujeres. Continúa dominando una segregación vertical de género, el habitual fenómeno del techo de cristal, como en otros aspectos de la carrera investigadora de las mujeres (Puy-Rodríguez, 2018). Los intentos por promocionar la igualdad de género en la investigación y su gestión por parte de la Comisión Europea y en los diferentes países, todavía parece lejos de dar los frutos apetecidos, desde luego, las revistas españolas de primera elección deben mejorar desde una perspectiva de igualdad de género.

\section{Tabla 4}

Distribución por género de las principales responsabilidades editoriales de las revistas editadas por el COP Madrid incluidas en el JCR/SSCI y SJR de 2018

\begin{tabular}{|c|c|c|c|c|c|}
\hline \multirow{2}{*}{ Revistas } & \multirow{2}{*}{ Editor/a-Director-a } & \multicolumn{2}{|c|}{ Editores/as Asociados/as } & \multicolumn{2}{|c|}{ Comités Editoriales } \\
\hline & & Hombres (\%) & Mujeres $(\%)$ & Hombres $(\%)$ & Mujeres (\%) \\
\hline APJ & Antonio Manzanero (UCM) & $2(33.33)$ & $4(66.67)$ & $21(55.26)$ & $17(44.74)$ \\
\hline cs & Ma Fé Rodriguez (UNED) & $5(71.43)$ & $2(28.57)$ & $39(63.93)$ & $22(36.07)$ \\
\hline EJPALC & Ramón Aree (USC) & $3(75)$ & $1(25)$ & $16(94.12)$ & $1(05.88)$ \\
\hline JWOP & Jesús F Salgado (USC) & $3(60)$ & $2(40)$ & $41(77.36)$ & $12(22.64)$ \\
\hline PEd & José A. León (UAM) & $7(70)$ & $3(30)$ & $23(47.92)$ & $25(52.08)$ \\
\hline PInt & $\begin{array}{l}\text { Enrique } \\
\text { Valencia) }\end{array}$ Gracia $\quad$ (U & $4(80)$ & $1(20)$ & $39(65)$ & $21(35)$ \\
\hline SJP & Javier Bandrés (UCM) & $10(62.5)$ & $6(37.5)$ & $19(90.48)$ & $2(09.52)$ \\
\hline Total & & $34(64.15)$ & $19(35.85)$ & $198(66.44)$ & $100(33.56)$ \\
\hline
\end{tabular}

Revistas: APJ (Anuario de Psicología Jurídica); CS (Clínica y Salud); JWOP (Journal of Work \& Organizational Psychology); PEd (Psicología Educativa); PInt (Psychosocial Intervention); SJP (Spanish Journal of Psychology); EJPALC (European Journal of Psychology Applied to Legal Context).

\section{Perfil de citas de las revistas en JCR/SSCI}

Las revistas editadas o coeditadas por el COP Madrid, incluidas en JCR/SSCI, muestran un perfil creciente, al contabilizar las citas desde todas las bases de datos de la WOS (Figura 2). Es más, los datos consolidados de 2019 permiten anticipar que las revistas van a tener más citas que en 2018, consolidándose o, incluso, mejorando su factor de impacto y su rango.

\section{Figura 2}

Evolución del número de citas de las revistas del COP Madrid, y de las revistas coeditadas desde la firma del convenio con el COP.

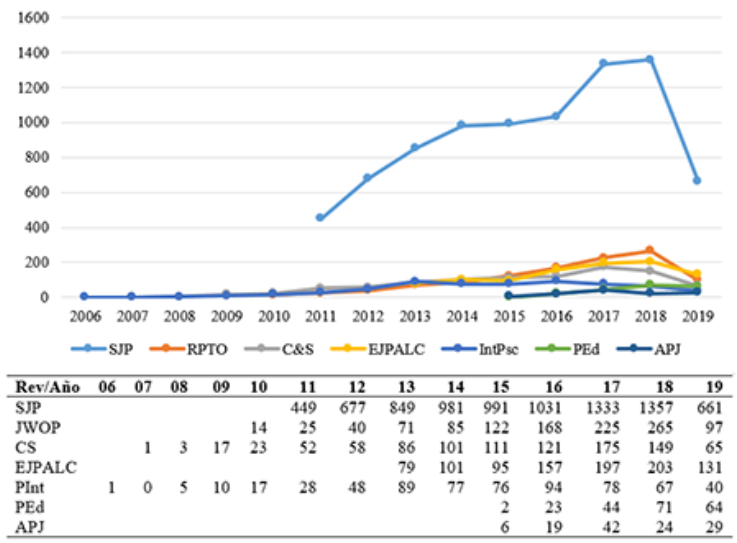

Nota. Acceso a datos de RPTO/JWOP 6/7/2019, 20,07; Acceso a datos de CS 6/7/2019, 20,26; Acceso a datos de APJ 6/7/2019, 20,46; Acceso a datos de PInt 6/7/2019, 21,06; Acceso a datos de PEd 6/7/2019, 21,26; Acceso a datos de SJP 6/7/2019, 21,46; Acceso a datos de EJPALC 6/7/2019, 22,06. Revistas: APJ (Anuario de Psicología Jurídica); CS (Clínica y Salud); JWOP (Journal of Work \& Organizational Psychology); PEd (Psicología Educativa); PInt (Psychosocial Intervention); SJP (Spanish Journal of Psychology); EJPALC (European Journal of Psychology Applied to Legal Context).

En el caso de las revistas coeditadas, tomando en consideración los datos solo desde la firma del convenio de coedición, encontramos un promedio de 925.11 en SJP y de 120.29 en EJPALC. Las cinco revistas producidas y editadas por el COP Madrid tienen los siguientes promedios: JWOP 111.3, CS 74, PInt 45, PEd 40.8 y APJ 34. 
Perfil de visitas y descargas de los contenidos de las revistas del COP Madrid

El número de visitas a la web colegial no ha cesado de incrementarse, hasta aproximarse a los tres millones (2 779 330) en 2018 (COP Madrid, 2019b). El incremento se ha producido en todas las áreas, aunque destacan la de revistas científicas, formación, y empleo. También, se ha venido incrementando la internacionalización de las visitas, sobre todo desde la Iberoamérica de habla española y desde EE. UU. y Reino Unido.

Desde que hay datos, se ha producido un fuerte incremento en el número de visitas a las revistas (que ha pasado de 75248 visitas en 2010, a 1 105962 en 2018). En paralelo, se ha producido también un incremento muy significativo en las descargas de los contenidos publicado en las revistas, pasando de 9495 descargas en 2010 a 102271 en 2018 (Figura 3).

El crecimiento en el número de visitas es exponencial. Se pasa, en apenas tres años de 75 248 a 388 731, en otros tres años a 935376 y en tres más a 1105 962, prácticamente un tercio de las visitas a la web colegial (COP Madrid, 2019b). Así mismo, se ha multiplicado casi por 11, en apenas 9 años, el número de descargas, desde 9 495 hasta 102271.

Los lectores más interesados por los contenidos de las revistas editadas por el COP Madrid son: España (40 \%), México (12\%), Perú (10 \%), Colombia (6 \%), Chile (5 \%), EE. UU. (4 \%) y Reino Unido (4 \%) (COP Madrid, 2013, p. 21). Los datos no son muy diferentes los siguientes años. Los lectores más interesados proceden, además de España (36 \%), México (10 \%), EE. UU. (9 \%), Portugal (7 \%), Perú (7 \%), Colombia (7 \%), Reino Unido (4 \%) y Argentina (4 \%) (COP Madrid, 2014, p. 19). Los lectores que, en 2015, más se interesan en los contenidos de las revistas, además de España (43 \%), proceden de países como México, EE. UU., Colombia, Argentina, Perú, Chile, Uruguay, Ecuador, Bolivia, Portugal, Reino Unido, Alemania, Costa Rica, India, etc.) (COP Madrid, 2016, p. 21).
En los últimos tres años, los datos continuarán siendo no solo positivos respecto del número de visitas y descargas de contenidos, sino también en relación con la descentralización respecto a los usuarios, con un aumento de la internacionalización de las revistas, llegando a conseguir visitas y descargas de 57 países. Si bien es cierto que el público español es siempre el lector con diferencia más numeroso, alrededor del $40 \%$, cada vez es mayor la progresión en el número de lectores de otros países como: México, EE. UU., Colombia, Argentina, Perú\#, Chile, Uruguay, Ecuador, Bolivia, Portugal, Reino Unido, Alemania, Costa Rica, China, Rusia, Australia e India, entre otros.

\section{Figura 3}

Resumen estadístico de visitas y descargas de revistas 2010-2018

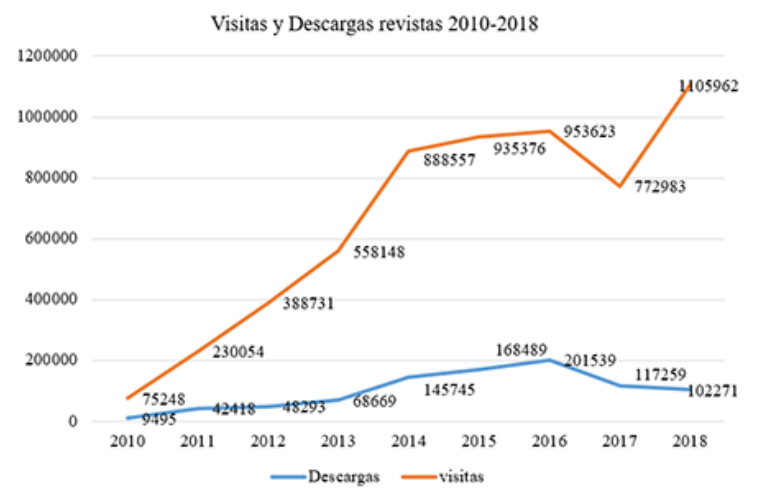

Un Colegio que empeñado en hacer accesibles sus revistas cada vez con mayor facilidad a sus potenciales usuarios. Las revistas del colegio estrenan, desde principios de 2018, nuevas webs para mejorar incluso los rigurosos requisitos de calidad editorial exigidos para mantenerse en la élite mundial, así como para satisfacer las expectativas de un número creciente de usuarios ya nativos digitales (Prensky, 2001a, 2001b). La remodelación de las webs alcanza a todos los aspectos y funcionalidades de las revistas, destacando: facilitar la lectura en los diferentes formatos en versión html, pdf y epub, hacerlas accesibles desde dispositivos móviles, satisfacer necesidades y demandas de los usuarios, permitir un acceso bilingüe en español e inglés y dar al usuario la posibilidad de compartir los artículos 
en redes sociales y exportarlos a los gestores de citas más usados. Se trata de un conjunto de cambios orientados a incrementar y mejorar la circulación y visibilidad de los artículos, los actuales y los de archivo, y de las revistas entre la comunidad científica (Guía del Psicólogo, 2018a, p. 18).

También en 2018, siguiendo el proyecto iniciado por el Colegio para aumentar su presencia en las redes sociales (Guía del Psicólogo, 2018b, p. 19), las revistas científico-profesionales del COP Madrid, además a través de la web del Colegio, en la sección publicaciones-revistas-científicoprofesionales, tienen cuentas en las redes sociales Facebook y Twitter (Guía del Psicólogo, 2018c, p. 10).

\section{Conclusiones}

En definitiva, el plan estratégico (Tabla 5) ha cumplido sus objetivos, y se ha renovado para seguir avanzando. Los datos confirman el éxito de la estrategia que venía desarrollando el COP Madrid para incrementar su visibilidad y el reconocimiento internacional de la producción científica en psicología, publicada en España. La estrategia había permitido lograr el incremento de visibilidad nacional e internacional pretendido y ayudar a visibilizar e internacionalizar la psicología, hecha sobre todo en España.
Tabla 5

Características de las revistas editadas por el COP

Madrid antes de 2009 y en la actualidad (adaptado de Salgado, 2015)

\begin{tabular}{|c|c|c|}
\hline Variable & Antes 2009 & En la actualidad \\
\hline Imagen de marea & No & $\begin{array}{l}\text { Sí, única en prácticamente todas las } \\
\text { revistas editadas, llega incluso a } \\
\text { alguna coeditada }\end{array}$ \\
\hline Tipologia de articulos & $\begin{array}{l}\text { Profesión, intervenciones, } \\
\text { noticias, comentarios,.... }\end{array}$ & Investigaciỏn, profesión \\
\hline Idioma & $\begin{array}{l}\text { Espantol, lenguas } \\
\text { cooficiales }\end{array}$ & Español, inglés, otros idiomas \\
\hline Fechas de Publicación & Irregular & $\begin{array}{l}\text { Anticipada (advanced online desde } \\
\text { 2015) }\end{array}$ \\
\hline Periodicidad & Irregular & $\begin{array}{l}\text { Normalizada (anual, semestral o } \\
\text { cuatrimestral) }\end{array}$ \\
\hline $\begin{array}{l}\text { Ajuste a criterios } \\
\text { Latindex }\end{array}$ & No & Si \\
\hline $\begin{array}{l}\text { Recepción electrónica } \\
\text { de manuscritos }\end{array}$ & No, irregular & Si, desde 2014 \\
\hline DOI & No & Sí, a partir de 2010 \\
\hline $\begin{array}{l}\text { Presencia de autores } \\
\text { no españoles }\end{array}$ & $\begin{array}{l}\text { Muy escasa, habitualmente } \\
\text { mediante invitación }\end{array}$ & $\mathrm{Si}$, cada vez más numerosa \\
\hline $\begin{array}{l}\text { Presencia en bases de } \\
\text { datos }\end{array}$ & $\begin{array}{l}\text { Irregular, sobre todo } \\
\text { españolas. Testimonial en } \\
\text { JCR o SJR }\end{array}$ & $\begin{array}{l}\text { Si, en bases de datos y de citas } \\
\text { internacionales (JCR, SJR, ...). } \\
\text { Institución española más } \\
\text { representada }\end{array}$ \\
\hline $\begin{array}{l}\text { Visibilidad } \\
\text { internacional }\end{array}$ & Escasa, Iberoamérica & Si, cada vez más global \\
\hline Factor de impacto & $\begin{array}{l}\text { No, salvo Psicothema. Solo } \\
\text { en IN-RECS (a partir de } \\
\text { revistas españolas) }\end{array}$ & $\mathrm{Si}$ \\
\hline $\begin{array}{l}\text { Ajuste a Criterios } \\
\text { FECYT y Agencias de } \\
\text { Calidad }\end{array}$ & No & Si, desde 2012 \\
\hline $\begin{array}{l}\text { Reconocimiento } \\
\text { institucional de calidad }\end{array}$ & No & $\begin{array}{l}\text { Todas. Sello de Calidad FECYT, } \\
\text { desde } 3 \text {." convocatoria } 2011^{\text {s. }}\end{array}$ \\
\hline
\end{tabular}

En apenas una década, el COP Madrid ha pasado de ninguna revista en JCR/SSCI de las cuatro entonces recogidas en la base de datos, a siete de las 17 actuales. De hecho, las cinco últimas revistas españolas de psicología incorporadas en JCR son editadas en solitario por el COP Madrid. Ha pasado de resaltar como noticia la inclusión de alguna de sus revistas en una o dos bases de datos internacionales, a circular habitualmente por más de 20 , de las consideradas por la comunidad científica como de referencia. La inclusión de las publicaciones del Colegio en bases de datos ha supuesto tanto el fortalecimiento de la comunicación científica española en Iberoamérica, y en el resto del mundo, como el reconocimiento de la calidad científica de los contenidos de las revistas que edita el Colegio y de una gestión editorial crecientemente profesionalizada.

Esto convierte al Colegio de Madrid en una institución de referencia en nuestro sector profesional, pero también académico. Es la entidad editora de entre los países de lengua hispano-portuguesa más importante, y más reconocida internacionalmente, a nivel cienti\#fico-profesional, superando a otros 
colegios de psicólogos, universidades, centros o asociaciones que también editan revistas de psicología de este carácter. En este sentido, como apunta Tortosa-Pérez et al. (2020), la presencia en diferentes bases de datos de las revistas editadas o coeditadas por el COP Madrid es mucho mayor que las editadas por otras instituciones, diferencias que resultaron ser significativas.

Si bien la estrategia seguida por el COP Madrid ha dado resultados con respecto al posicionamiento internacional de sus revistas, cabe plantear la necesidad de aplicar, dentro de esta estrategia, una perspectiva de género, procurando la igualdad en sus órganos directivos.

\section{Agradecimientos}

Los autores quieren mostrar su agradecimiento a Ana Isabel García Colmenarejo (Departamento de Publicaciones. Colegio Oficial de Psicólogos de Madrid) y a Jesús F. Salgado (Universidad de Santiago de Compostela, y editor en jefe de la revista Journal of Work and Organizational Psychology), por su inestimable, desinteresada y constructiva colaboración.

\section{Referencias}

Anónimo (1995). Editorial. Psicología Educativa, 1(1). Recuperado de https://journals.copma drid.org/psed/art/471c75ee6643a10934502 bdafee $198 \mathrm{fb}$

Aznar, M., \& Brox, V. (2010). Editorial. Clínica Contemporánea, 1(1), 3. Recuperado de https://www.revistaclinicacontemporan ea.org/archivos/cc2010v1n1a1.pdf

Bucchi, M. (2004). Science in Society. Nueva York: Routledge.

Camarero, C. (1989). Memoria escrita [Resumen]. Papeles del Psicólogo, 36-37, 64-67. Recuperado de http://www.papelesd elpsicologo.es/resumen?pii $=378$

Carpintero, H. (1980). La psicología actual desde una perspectiva bibliométrica: una introducción. Análisis y Modificación de
Conducta, 11-12, 9-23. https://doi.org/10.3 3776/amc.v6i11-12.1541

Carpintero, H. (Ed.). (1983a). Historia y teoría psicológica. Valencia: Alfaplus.

Carpintero, H. (1983b). Algunos métodos cuantitativos en historia de la ciencia psicológica. Millars, 8(2), 49-60.

Carpintero, H., \& Peiró, J. M. (1981). Psicología contemporánea. Teoría y métodos cuantitativos para el estudio de su literatura científica. Valencia: Alfaplus.

Casas, F. (1992). Editorial. Intervención Psicosocial, 1(1). Recuperado de https://jour nals.copmadrid.org/pi/art/d25414405eb37 dae1c14b18d6a2cac34

Civera, C., \& Alonso, F. (1995). La evolución de la Psicología profesional en España. Revista de Historia de la Psicología, 16(3-4), 281-291. Recuperado de https://www.revistahistoriapsicologia.es/ revista/1995-vol-16-n\%C3\%BAm-3-4/

Civera, C., \& Tejero, P. (1993). Una perspectiva de la Psicología profesional en los "80". El colegio oficial de psicólogos y sus publicaciones. Revista de Psicología. Universitas Tarraconensis, 15(2), 107-127.

Colegio Oficial de Psicólogos. Delegación Madrid. (1982). Memoria 1981. Madrid: Autor. Recuperado de https://www.copmad rid.org/web/img_db/publicaciones/memori a-actividades-1981-5cc2f73ad64e6.pdf

Colegio Oficial de Psicólogos de Madrid. (1997). Memoria de actividades 1996. Madrid: Autor. Recuperado de https://www.copmadrid.org/web/img_db /publicaciones/memoria-1996-5c34efe07cc 9c.pdf

Colegio Oficial de Psicólogos de Madrid. (1998). Memoria de actividades 1997. Madrid: Autor. Recuperado de https:/www.copmadrid.org/web/img_db /publicaciones/memoria-1997-5c34f1199e 4dd.pdf

Colegio Oficial de Psicólogos de Madrid. (1999). Memoria de actividades 1998. Madrid: Autor. Recuperado de. https://www.copmadrid.org/web/img_d b/publicaciones/memoria-1998.pdf 
Colegio Oficial de Psicólogos de Madrid. (2000). Memoria de actividades 1999. Madrid: Autor. Recuperado de https:/www.copmadrid.org/web/img_db /publicaciones/memoria-1998.pdf

Colegio Oficial de Psicólogos de Madrid. (2002). Memoria de actividades 2001. Madrid: Autor. Recuperado de https://www.copmadrid.org/web/img_db /publicaciones/memoria-2001.pdf

Colegio Oficial de Psicólogos de Madrid. (2004). Memoria de actividades 2003. Madrid: Autor. Recuperado de https://www.copmadrid.org/web/img_db /publicaciones/memoria-2003.pdf

Colegio Oficial de Psicólogos de Madrid. (2007). Memoria 2006. Madrid: Autor. Recuperado de https://www.copmadrid.org/web/img_db /publicaciones/memoria-2006.pdf

Colegio Oficial de Psicólogos de Madrid. (2010). Memoria 2009. Madrid: Autor. Recuperado de https:/www.copmadrid.org/web/img_db /publicaciones/memoria-2009.pdf

Colegio Oficial de Psicólogos de Madrid. (2011). Memoria 2010. Madrid: Autor. Recuperado de https://www.copmadrid.org/web/img_db /publicaciones/memoria-2010.pdf

Colegio Oficial de Psicólogos de Madrid. (2012). Memoria 2011. Madrid: Autor. Recuperado de https://www.copmadrid.org/web/img_db /publicaciones/memoria-2011.pdf

Colegio Oficial de Psicólogos de Madrid. (2013). Memoria 2012. Madrid: Autor. Recuperado de https://www.copmadrid.org/web/img_db /publicaciones/memoria-2012.pdf

Colegio Oficial de Psicólogos de Madrid. (2014). Memoria 2013. Madrid: Autor. Recuperado de https://www.copmadrid.org/web/img_db /publicaciones/memoria-2013-5575ca86db 2d5.pdf

Colegio Oficial de Psicólogos de Madrid. (2016). Memoria anual 2015. Madrid: Autor. Recuperado de https://www.copmadrid.org/web/img_db /publicaciones/memoria-2015.pdf

Colegio Oficial de Psicólogos de Madrid. (2017). Memoria 16. Madrid: Autor. Recuperado de https://www.copmadrid.org/web/img_db /publicaciones/memoria-2016.pdf

Colegio Oficial de Psicólogos de Madrid. (2019a). Historia del Colegio. Madrid: Autor. Recuperado de https://www.copmadrid.org /web/el-colegio/historia-colegio

Colegio Oficial de Psicólogos de Madrid. (2019b). Memoria de actividades 2018. Madrid: Autor. Recuperado de https://www.copmadrid.org/web/img_db /publicaciones/memoria-actividades-20185c94e0d6ec449.pdf

Consejería de Presidencia. Comunidad de Madrid. Decreto 1 de 2001. Por el que se constituye, por segregación, el Colegio Oficial de Psicólogos de Madrid. Enero 11 de 2001. BOE de 18 de enero de 2001. Recuperado de http://www.copmadrid.org/webcopm/re cursos/2001decreto11-2001-01.pdf

Fernández, L., \& Rodríguez, J. (2014). The "impact factor style of thinking": A new theoretical framework. International Journal of Clinical and Health Psychology, 14(2), 154-160. https://doi.org/10.1016/S1697-26 $00(14) 70049-3$

García-Naveira, A. (2016). Editorial. Revista de Psicología Aplicada al Deport y al Ejercicio Físico, 1(1), e1, 1. https://doi.org/10.5093/r padef2016a1

Giménez, E., Rodríguez, G., \& de la Moneda, M. (2009). Spanish scientific journals on psychology (II): editorial quality, visibility, internationality and editor's attitude towards open access. Psychology Science Quarterly, 51(1), 119-134. Recuperado de http://digital.csic.es/bitstream/10261/23 606/1/PSQ_Gimenez-Toledo_2009.pdf

González-Sala, F., \& Osca-Lluch, J. (2018). Desigualdad de género en órganos directivos y producción científica de las revistas iberoamericanas de psicología de mayor visibilidad internacional. Revista Española de Documentación Científica, 41(3), e211. https://doi.org/10.3989/redc.2 018.3 .1506

González-Sala, F., Osca-Lluch, J., \& HabaOsca, J. (2019). Information resources: 
Differential characteristics between IberoAmerican and Dutch JCR psychology Journals from 1998 to 2017. Resources, 8(111), 1-18. https://doi.org/10.3390/resou rces8020111

Guía del Psicólogo. (2018a). Las revistas del Colegio en las redes sociales. Guía del Psicólogo, 389, 18. Recuperado de https://www.copmadrid.org/web/img_db /publicaciones/gp201803-pdf-5abbbeffbe94 e.pdf

Guía del Psicólogo. (2018b). El Colegio aumenta su presencia en las redes sociales. Guía del Psicólogo, 395, 19. Recuperado de https://www.copmadrid.org/web/img_db /publicaciones/guia-del-psicologo-2018-vol -nov-n-396-5bd8aa67f2192.pdf

Guía del Psicólogo. (2018c). Las revistas del Colegio en las redes sociales. Guía del Psicólogo, 396, 10. Recuperado de https://www.copmadrid.org/web/img_db /publicaciones/guia-del-psicologo-2018-vol -nov-n-397-5bffd9c7dd2e7.pdf

Hernández-Gordillo, A. (1989). El modelo organizativo del COP [Resumen]. Papeles del Psicólogo, 36-37. Recuperado de http://www.papelesdelpsicologo.es/resu men?pii $=374$

Hernández-Gordillo, A. (1995). Editorial (II). Psicología del Trabajo y Organizaciones, 11(31), 7. Recuperado de https://journals.copmadrid.org/jwop/art/ d2ed45a52bc0edfa11c2064e9edee8bf

Jefatura del Estado. Ley 43 de 1979. Creación del Colegio Oficial de Psicólogos. Diciembre 31 de 1979. BOE n. ${ }^{\circ} 7$, de 8 de enero de 1980. Recuperado de https://www.boe.es/buscar/ act.php?id=BOE-A-1980-405

Jiménez-Contreras, E., Robinson-García, N., \& Cabezas-Clavijo, A. (2011). Productividad e impacto de los investigadores españoles: umbrales de referencia por áreas científicas. Revista Española de Documentación Cientifica, 34, 505-526. https://doi.org/10.3 989/redc.2011.4.828

Junta de Gobierno. (1981). El Colegio y la Delegación de Madrid [Resumen]. Papeles del Psicólogo, 0. Recuperado de http://www. papelesdelpsicologo.es/resumen?pii $=18$

Junta de Gobierno. (1981b). Un año como punto de partida. Papeles del Psicólogo, 0, 1-2 Recuperado de http://www.papelesdelpsico logo.es/resumen?pii=1

Junta General. Delegación de Madrid. (1991). Memoria balance de situación pérdidas y ganancias 1990. Plan de actividades presupuesto 1991. Madrid: COP Madrid. Recuperado de https://www.copmadrid.org /web/publicaciones/memorias-anteriores

Knorr-Cetina, K., \& Mulkay, M. (1983). Introducction: Emerging principles in social studies of science. En K. Knorr-Cetina \& M. Mulkay, Science observed. Perspectives on the social studies of science (pp. 1-17). Londres: Sage.

Larivière, V., Haustein, S., \& Mongeon, P. (2015). The oligopoly of academic publishers in the Digital Era. PLoS ONE, 10, e0127502. http s://doi.org/10.1371/journal.pone.0127502

Martínez, J. (2001). El COP de Madrid: un Colegio con historia [Resumen]. Papeles del Psicólogo, 80, 54-59. Recuperado de http://www.papelesdelpsicologo.es/resu men?pii $=875$

Ministerio de Educación y Cultura. Real Decreto 481 de 1999. Por el que se aprueban los Estatutos Generales del Colegio Oficial de Psicólogos. Marzo 18 de 1999. BOE n. 83, de 7 de abril de 1999. Recuperado de https://www.boe.es/buscar/pdf/1999/BO E-A-1999-7734-consolidado.pdf

Ministerio de Educación, Cultura y Deporte. Real Decreto 1902 de 2000. Por el que se acuerda la segregación de las Delegaciones del Colegio Oficial de Psicólogos. Noviembre 20 de 2000. BOE, n. ${ }^{\circ} 291$, de 5 de diciembre de 2000. Recuperado de https://boe.es/boe/dias/200 0/12/05/pdfs/A42582-42583.pdf

Ministerio de Universidades e Investigación. Orden de 1980. Por la que se aprueban los Estatutos Provisionales del Colegio Oficial de Psicólogos. Marzo 24 de 1980. BOE, n. - 90, de 14 de abril de 1980. Recuperado 
de https://www.boe.es/eli/es/o/1980/03/24/ (2)/dof/spa/pdf

Munduate, M. (1985). Editorial: El enfoque sistémico del enriquecimiento del trabajo. Revista de Psicología del Trabajo y de las Organizaciones, 1(1), 4-17. Recuperado de https://journals.copmadrid.org/jwop/art/ 99c5e07b4d5de9d18c350cdf64c5aa3d

Osca-Lluch, J., González-Sala, F., Haba-Osca, J., Tortosa, F., \& Peñaranda-Ortega, M. (2019). Comunicación científica o cualificación para una Carrera académica: ¿Qué uso tienen los artículos en las revistas de psicología? Anales de Psicología, 35(1), 166-174. https://doi.org/10.6018/analesps. 35.1.329211

Osca-Lluch, J., González-Sala, F., Fonseca, S., \& Civera, C. (2017). Historia de la investigación española en psicología vista a través de las revistas científicas recogidas en la Web of Science durante el periodo 2003-2012. Universitas Psychologica, 16(2), 1-10. https://doi.org/10.11144/Javeriana.up sy16-2.hiep

Pérez, F. (2008). El Colegio Oficial de Psicólogos como productor de revistas científicas. Revista de Historia de la Psicología, 29(3-4), 171-183. Recuperado de https://www.revistahistoriapsicologia.es/ revista/2008-vol-29-n\%C3\%BAm-3-4/

Prensky, M. (2001a). Digital natives, digital inmigrants. Part 1. On the horizon, 9(5), 1-6. https://doi.org/10.1108/107481201104 24816

Prensky, M. (2001b). Digital natives, digital inmigrants. Part 2: Do they really think differently? On the horizon, 9(6), 1-6. https: //doi.org/10.1108/10748120110424843

Puy-Rodríguez, A. (2018). Introducción. En Científicas en cifras 2017. Estadística e indicadores de la (des)igualdad de género en la formación y profesión científica (pp. 22-37). Madrid: Ministerio de Ciencia, Innovación y Universidades. Recuperado de https://icono.fecyt.es/sites/default/files/fi lepublicaciones/cientificas_en_cifras_2017 .pdf
Ramírez, D. (1991). 1096-91: Los primeros cinco años de la sección de psicología jurídica. Anuario de Psicología Jurídica, 1(1), 9-15. Recuperado de https://journals.copmadrid. org/apj/archivos/1991/Arti1.htm

Ruiz, R., Delgado, E., \& Jiménez, E. (2006). Criterios del Institute for Scientific Information para la selección de revistas científicas. Su aplicación a las revistas españolas: metodología e indicadores. International Journal of Clinical and Health Psychology, 6(2), 401-424. Recuperado de http://www.aepc.es/ijchp/articulos_pdf/i jchp-184.pdf

Salgado, J. (octubre, 2015). La publicación de revistas científicas de psicología: la experiencia del Colegio Oficial de Psicólogos de Madrid. Trabajo presentado en I Jornadas Internacionales de Revistas de Ciencias de la Salud, Universidad Miguel Hernández, Valencia, Elche. Recuperado de http://jircs.umh.es/files/2015/10/Jesu\%C 3\%ACs-Salgado.pdf

Santolaya, F. (2001). Colegio y profesión [Resumen]. Papeles del Psicólogo, 80, 71-75. Recuperado de http://www.papelesdelpsico logo.es/resumen?pii=879

Tortosa-Pérez, M., González-Sala, F. E., Santolaya-Prego de Oliver, J., \& AguilarBustamante, C. (2020). El papel de la organización colegial-COP en el posicionamiento internacional de la psicología española (1979-2018). Anales de Psicología, 36(1), 12-23. https://doi.org/10.6 018/analesps.38869

Tortosa-Pérez, M., Osca-Lluch, J., AlfaroFerreres, E., \& López-López, W. (2019). Internacionalización y posicionamiento de las revistas espa olas de psicología. Anales de Psicología, 35(2), 332-340. https:// doi.org/10.6018/analesps.35.2.332171

\section{Notas}

* Artículo de revisión. 